\title{
Efficiently feeding single-mode fiber photonic spectrographs with an extreme adaptive optics system: on-sky characterization and preliminary spectroscopy
}

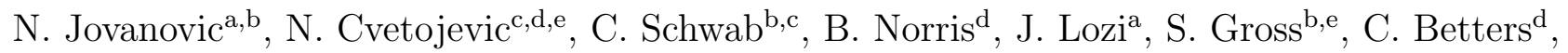 \\ G. Singh ${ }^{\mathrm{f}}$, O. Guyon ${ }^{\mathrm{a}, \mathrm{g}, \mathrm{h}, \mathrm{i}}$, F. Martinache ${ }^{\mathrm{j}}$, D. Doughty ${ }^{\mathrm{a}, \mathrm{h}}$, and P. Tuthill ${ }^{\mathrm{d}}$ \\ ${ }^{a}$ National Astronomical Observatory of Japan, Subaru Telescope, 650 North A'Ohoku Place, \\ Hilo, HI, 96720, U.S.A. \\ ${ }^{\mathrm{b}}$ Department of Physics and Astronomy, Macquarie University, NSW 2109, Australia \\ cAustralian Astronomical Observatory, 105 Delhi Rd, North Ryde NSW 2113, Australia \\ ${ }^{\mathrm{d} S y d n e y ~ I n s t i t u t e ~ f o r ~ A s t r o n o m y ~(S I f A), ~ I n s t i t u t e ~ f o r ~ P h o t o n i c s ~ a n d ~ O p t i c a l ~ S c i e n c e ~(I P O S), ~}$ \\ School of Physics, University of Sydney, NSW 2006, Australia \\ ${ }^{\mathrm{e}}$ Centre for Ultrahigh-bandwidth Devices for Optical Systems (CUDOS) \\ ${ }^{\mathrm{f} J e t}$ Propulsion Laboratory, 4800 Oak Grove Drive, MS 183-901, Pasadena, CA 91109 \\ ${ }^{\text {g} S t e w a r d ~ O b s e r v a t o r y, ~ U n i v e r s i t y ~ o f ~ A r i z o n a, ~ T u c s o n, ~ A Z, ~ 85721, ~ U . S . A . ~}$ \\ ${ }^{\mathrm{h}}$ College of Optical Sciences, University of Arizona, Tucson, AZ 85721, U.S.A. \\ ${ }^{i}$ Astrobiology Center of NINS, 2-21-1, Osawa, Mitaka, Tokyo, 181-8588, Japan \\ ${ }^{j}$ Observatoire de la Cote d'Azur, Boulevard de l'Observatoire, Nice, 06304, France
}

\begin{abstract}
High-order wavefront correction is not only beneficial for high-contrast imaging, but also spectroscopy. The size of a spectrograph can be decoupled from the size of the telescope aperture by moving to the diffraction limit which has strong implications for ELT based instrument design. Here we present the construction and characterization of an extremely efficient single-mode fiber feed behind an extreme adaptive optics system (SCExAO). We show that this feed can indeed be utilized to great success by photonic-based spectrographs. We present metrics to quantify the system performance and some preliminary spectra delivered by the compact spectrograph.
\end{abstract}

Keywords: Extreme AO, Photonic instruments, Fiber injection, Arrayed Waveguide Gratings, Diffractionlimited performance, Pupil apodization, Spectroscopy

\section{INTRODUCTION}

Photonic technologies are now ubiquitous in everyday life. These technologies offer a diverse range of functionality including spectral filtering, ${ }^{1}$ dispersion, ${ }^{2}$ spatial filtering ${ }^{3}$ and reformatting ${ }^{4}$ to name a few, all in a compact and robust footprint which can be easily stabilized. This makes these devices highly desirable for implementing into astronomical instrumentation.

However, these functionalities are only available if the device is designed to be diffraction-limited. This means that the device operates with only a single-mode which is characterized by a Gaussian intensity distribution and a flat wavefront (i.e. light can only propagate through the device if it is in this mode). Coupling light from a telescope into a diffraction-limited device of this nature efficiently is where the difficulty lies.

The point-spread function (PSF) at the focal plane of a telescope is distorted by atmospheric turbulence and is therefore far from diffraction-limited. Adaptive optics (AO) systems are commonly used to correct for this and go someway to restoring the PSF by flattening the wavefront. However, typical single conjugate AO systems used at many observatory's offer Strehl ratios of the order of $20-50 \%$ in the H-band. ${ }^{5,6}$ This translates to 200-320 nm of RMS wavefront error at a wavelength of $1600 \mathrm{~nm}$. Still far from the ideal flat wavefront $(0 \mathrm{~nm}$

Nemanja Jovanovic: E-mail: jovanovic.nem@gmail.com, Telephone: 18089345959

Ground-based and Airborne Instrumentation for Astronomy VI, edited by Christopher J. Evans, Luc Simard, Hideki Takami Proc. of SPIE Vol. 9908, 99080R · (c) 2016 SPIE · CCC code: 0277-786X/16/\$18 · doi: 10.1117/12.2234299 
RMS error). In addition, the PSF of a telescope is an Airy pattern produced by the uniform illumination of the telescope optics which creates diffraction rings around the PSF. This means that the PSF intensity distribution is also mismatched with respect to the ideal Gaussian shape desired by photonic devices. It is these mismatches in wavefront and intensity that make it intrinsically difficult to couple light efficiently into single-mode devices from ground-based telescopes and hence exploit photonic functionalities.

Combating these two issues involves the use of advanced wavefront control systems termed Extreme AO systems (ExAO) as well as pupil apodization techniques common place in coronagraphy. ExAO systems enabling $\sim 90 \%$ Strehl ratios in the H-band are now operational at several observatory's. ${ }^{7,8}$ With this level of wavefront correction the residual wavefront error is reduced to $\sim 80 \mathrm{~nm}$ RMS at a wavelength of $1600 \mathrm{~nm}$. This is achieved by controlling a larger region around the PSF in the focal plane and doing so at higher speed then with conventional AO systems.

Pupil apodization is the method whereby the intensity distribution in the pupil plane (and hence the focal plane as they are correlated) is remapped such that it has a gradually degrading signal that disappears at the edges of the pupil, which does not create diffraction features in the focal plane. A lossless form of apodization that has been termed Phase Induced Amplitude Apodization (PIAA), ${ }^{9}$ relies on the use of two aspheric optics positioned in the pupil plane to reformat the beam. This was originally designed for coronagraphy where the aim is to cancel the on-axis star light and allow a closely separated faint companion to be transmitted by the coronagraph. Indeed the requirements for optimum cancellation are identical to efficient fiber injection: the intensity distribution should be as compact as possible with no diffraction features.

In this body of work we present our recent results in our endeavor to address these limitations. We will outline the fiber injection system that is deployed on the Subaru Coronagraphic Extreme Adaptive Optics (SCExAO) instrument and both the laboratory and on-sky characterization of the efficiency with which we can couple light into a single-mode fiber (SMF). Subsequent to this we demonstrate the injection of this light into a compact photonic spectrograph realizing a highly efficient instrument.

\section{THE INSTRUMENT AND FIBER INJECTION}

\subsection{The Subaru Coronagraphic Extreme Adaptive Optics instrument}

A schematic of the SCExAO instrument is shown in Fig. 1. A complete description of the instrument is offered elsewhere. ${ }^{10}$ Here we focus on the main elements relevant to this work. The main instrument consists of two optical benches mounted on top of each other connected via a periscope. The light enters the bottom bench (Infrared (IR) bench) from the facility AO system, AO188, ${ }^{6}$ which does a correction upstream of SCExAO. In median seeing AO188 delivers a beam with $30-40 \%$ Strehl ratio in the H-band. The visible light is reflected at the dichroic and sent up the periscope to the bench where the pyramid wavefront sensor is located (visible bench). The pyramid wavefront sensor is the primary wavefront sensor in SCExAO and operates at 1.5-3.5 kHz with very low latency $(\sim 1 \mathrm{~ms})$. It is used to drive the ExAO loop because of its exquisite sensitivity to high order modes. The correction is applied to the 2000-element deformable mirror (DM) (Bostom MicroMachines Corporation) on the bottom bench common to all beam paths. After the DM, the light passes through a common pupil mask which simulates the spiders and secondary of the telescope. Note on-sky SCExAO observes in fixed pupil mode and the pupil is oriented with the k-mirror in AO188 so that it sits behind the internal pupil mask. The light is then passed through the PIAA optics described in the introduction. A pair of aspheric lenses made from $\mathrm{CaFl}_{2}$ are used to do the apodization. The first lens moves some of the rays of light radially from the edge of the beam towards the center while the second lens recollimates the beam. ${ }^{11}$ This creates a beam with a prolate spheroid cross-sectional profile (near-Gaussian) and a $1 / e^{2}$ beam size of $7.9 \mathrm{~mm}$. The beam is then focused by a $519 \mathrm{~mm}$ off-axis parabola (OAP) (OAP2 in Fig. 1). The f65 beam is intercepted on the way to the focus by a deployable $1180 \mathrm{~nm}$ short pass filter (>95\% reflectivity from $1200-1750 \mathrm{~nm}$ ). The filter steers the light in band towards the fiber injection rig as shown in Fig. 2.

\subsection{Fiber injection}

The rig consists of a 5-axis stage mounted on two parallel rails. The X, Y \& Z axes are driven by linear stepper motors (T-NA08A25, Zaber Technologies) with a minimum step size of $50 \mathrm{~nm}$. The tip/tilt axes have hand driven 

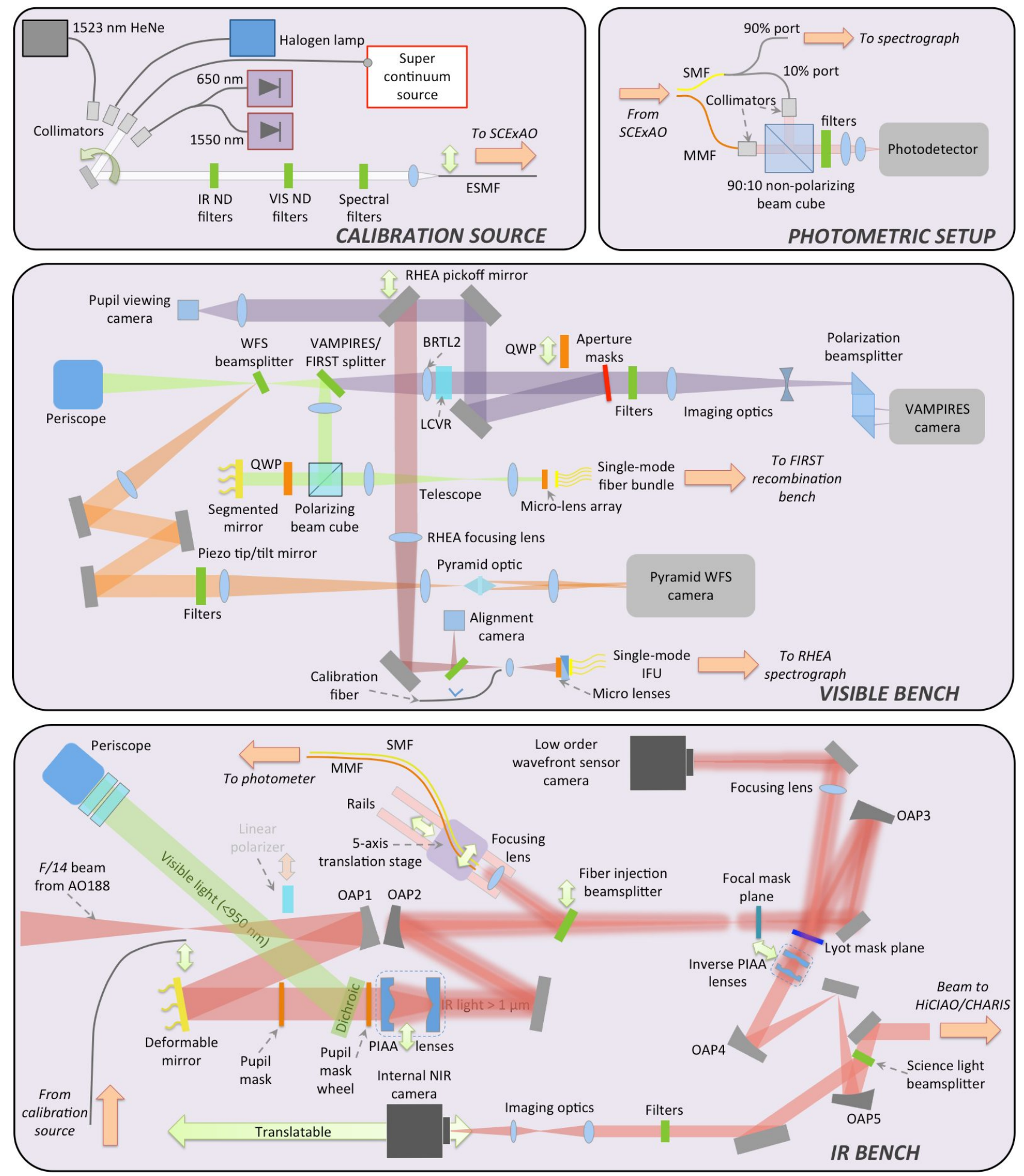

Figure 1. Schematic of the SCExAO instrument. (Top left) The calibration source which allows the user to select between a super continuum source, $1523 \mathrm{~nm}$ HeNe laser, 650/1550 nm laser diode and a halogen lamp. (Top right) The photometric setup for calibrating the coupling efficiency. (Middle) Visible bench of SCExAO which hosts the pyramid wavefront sensor. (Bottom) Infrared bench of SCExAO which hosts the fiber injection, the deformable mirror and the PIAA lenses. 


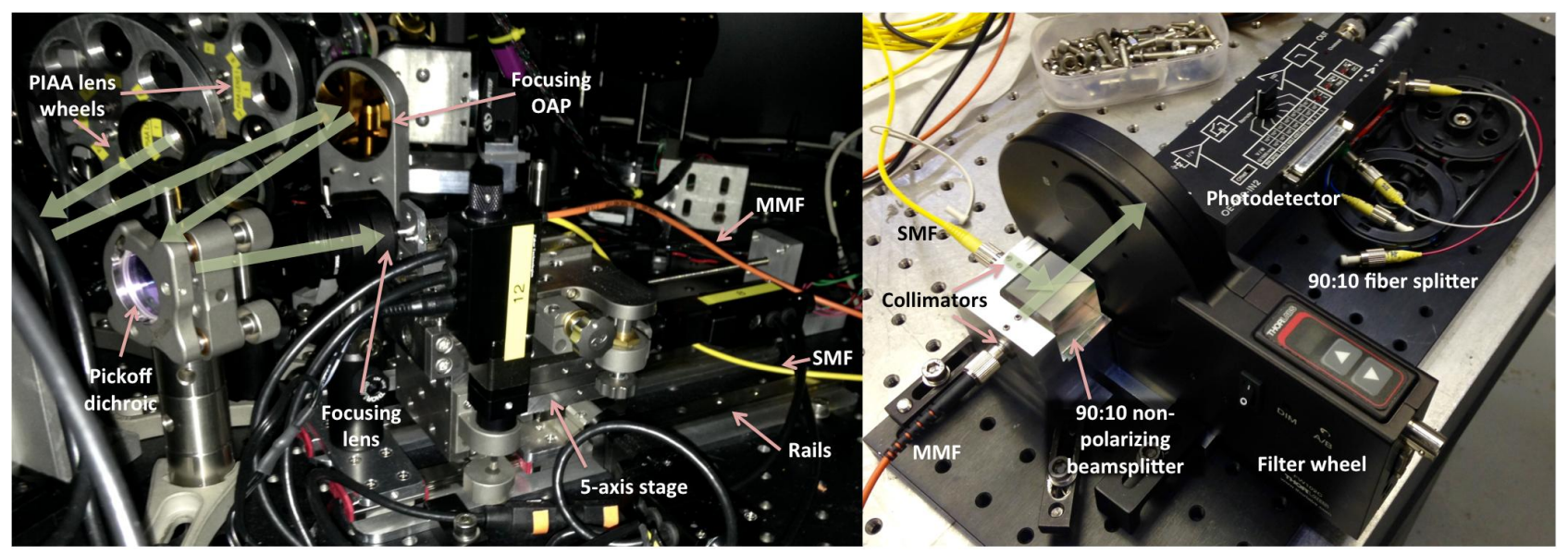

Figure 2. (Left) Image of the fiber injection mechanics. (Right) Image of the photometric setup. Transparent green arrows indicate the direction of the light path.

actuators. A bracket which can support several fibers is mounted atop the 5-axis stage. For these experiments the bracket held a single-mode fiber (SMF-28-J9) and a $365 \mu \mathrm{m}$ core diameter, step index multimode fiber (MMF) (FG365LEC). The purpose of the MMF will be explained below. Both were terminated with SMA connectors for ease of mounting. Only one fiber was aligned at the focus at any one time. To swap between them the stage was translated horizontally/vertically to bring the other fiber into the focus.

A small aspheric lens $(f=7.5 \mathrm{~mm}, \phi=5.0 \mathrm{~mm})$ was placed in front of the rig, on a separate carriage also mounted on the rails. The purpose of the lens was to adjust the focal ratio of the beam being injected into the fiber and hence optimize the coupling. To adjust the focal ratio, both the fiber rig and the small lens were moved along the rails with the aid of a stepper motor. As the focal ratio was changed, the coupling into the fiber was reoptimized (X, Y \& Z). The light was first coupled into the large MMF for simplicity. A blind step of the appropriate size was used to move the SMF fiber into the focus. In order to fine tune its alignment, visible laser light was reverse injected into the bench through the SMF. The fiber rig was adjusted laterally and in tip/tilt until the reverse injected beam was co-aligned with the incoming beam and it was centered on all optics upstream to the injection. At this point, light could be coupled into the SMF in the forwards direction. An optimization was carried out by scanning the stage in X, Y \& Z.

The optimum focal ratio for injection is the one that creates a PSF which has the best overlap with the mode of the fiber. The SMF-28-J9 has a $10.4 \mu \mathrm{m}, 1 / e^{2}$ mode field diameter and it was determined from simulation, a focal ratio of $55.3 \pm 0.1$ is required for optimally coupling in the apodized beam. The fiber rig and aspheric lens were scanned along the length of the rails and the coupling into the SMF was optimized at each position. From this the position of maximum coupling and hence the optimum focal ratio was located and the rig was parked at this location.

\subsection{Photometric setup}

To determine the coupling efficiency both on and off sky the light coupled into the SMF and MMF was transported to a photometric setup. The large extent of the MMF $(\sim 1.4$ " on-sky) enabled it to collect all of the flux in the focal plane regardless of the level of $\mathrm{AO}$ correction. This was used as a reference to calibrate the flux in the SMF and hence the coupling efficiency. For clarity, the process involved first measuring the flux through the SMF followed by the flux through the MMF for the same target under the same conditions, and using this to determine the coupling efficiency.

To measure the flux through each fiber, their outputs were reimaged onto a single sensitive photodetector (OE-200-IN2, Femto) with the aid of fiber collimators and a lens pair as shown in Fig. 1 and Fig. 2. A $90: 10$ non-polarizing beam cube was used to get both beams onto the detector (note this means that $90 \%$ of the flux from the MMF was thrown away and $10 \%$ from the SMF). The system was aligned so that both of the spots from the SMF and MMF landed on the $300 \mu \mathrm{m}$ diameter, InGaAs photodiode. Recall that only one of the fibers 

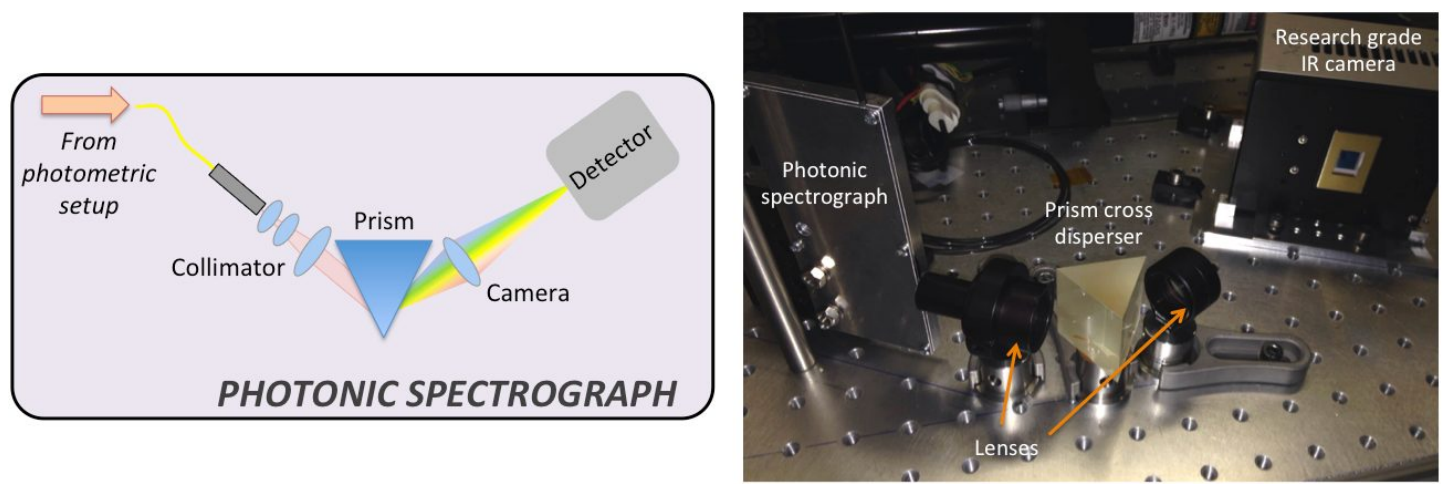

Figure 3. (Left) Schematic of the photonic spectrograph (Right) As built image of the photonic spectrograph.

is in the focus of the beam at any one time and so only a single beam is ever on the detector at once. A filter wheel was used to measure the coupling into the SMF in six channels across the J and H-bands (1250, 1300, 1350, 1500, 1550, and $1600 \mathrm{~nm}$ ). All had a $25 \mathrm{~nm}$ bandwidth except for the $1600 \mathrm{~nm}$ one which had a $50 \mathrm{~nm}$ bandwidth.

\subsection{Photonic spectrograph}

The light from the SMF was split with a $90: 10$ ratio with an achromatic fiber splitter (TW1550R2F2, Thorlabs) before being injected into the photometric setup (see Fig. 1). The $10 \%$ port was connected to the photometric setup while the $90 \%$ port was fed to a photonic spectrograph.

The photonic spectrograph is shown in Fig. 3. The light delivered from the photometric setup was first injected into an arrayed waveguide grating (AWG) based spectrograph. This compact photonic device has a resolving power of $R=7000$, has a free spectral range of $52 \mathrm{~nm}$, and has been the topic of numerous previous studies. $^{12,13}$ To separate the orders, the output beam was cross-dispersed onto a detector. The light was first collimated by $3, f=50 \mathrm{~mm}$ achromats, before being dispersed by a NSF11 prism. The output beam was focused with a $f=150 \mathrm{~mm}$ achromat onto an InGasAs detector (Xeva-1.7-320 TE3, Xenics). The $320 \times 256$ pixel camera was inclined at $45^{\circ}$ with respect to the beam for optimum focus of the spectra at all wavelengths simultaneously. The overall system was designed to have a resolving power between 4500-6000 in J and $\mathrm{H}$ bands.

\subsection{Calibration of spectra}

To calibrate the spectra, a flat field was taken with the halogen lamp in the calibration source (see Fig. 1) injected into SCExAO and through all subsequent optics to the detector of the spectrograph. As the resolving power was relatively low, a simple wavelength calibration was implemented with the use of the HeNe laser centered at $1523 \mathrm{~nm}$ in the calibration source.

\section{RESULTS}

\subsection{SMF coupling efficiency}

The coupling efficiency was characterized off-sky with varying degrees of wavefront error by using the turbulence simulator. In this case the super continuum source was used in conjunction with phase maps applied to the DM. The phase maps were based on a Kolmogorov power spectrum with the low spatial frequencies attenuated to mimic a low order AO correction. The phase map was continuously passed across the DM at a rate which corresponds to a windspeed of $10 \mathrm{~m} / \mathrm{s}$. To accurately determine the applied wavefront error, images of the PSF were taken in the H-band with the internal NIR camera. The images were dark subtracted and then the Strehl ratio was calculated for a cube of 1000 images and averaged. The coupling efficiency as a function of the Strehl ratio is shown in the left panel of Fig. 4. Using Marchels approximation the applied RMS wavefront error was determined from the measured Strehl ratio. The coupling efficiency as a function of the applied RMS wavefront error is shown in the right panel of Fig. 4. It can be seen that the coupling efficiency is maximum at longer wavelengths $(1500-1600 \mathrm{~nm})$ and reaches a value as high as $74 \%$ in the limit where the wavefront error is low 

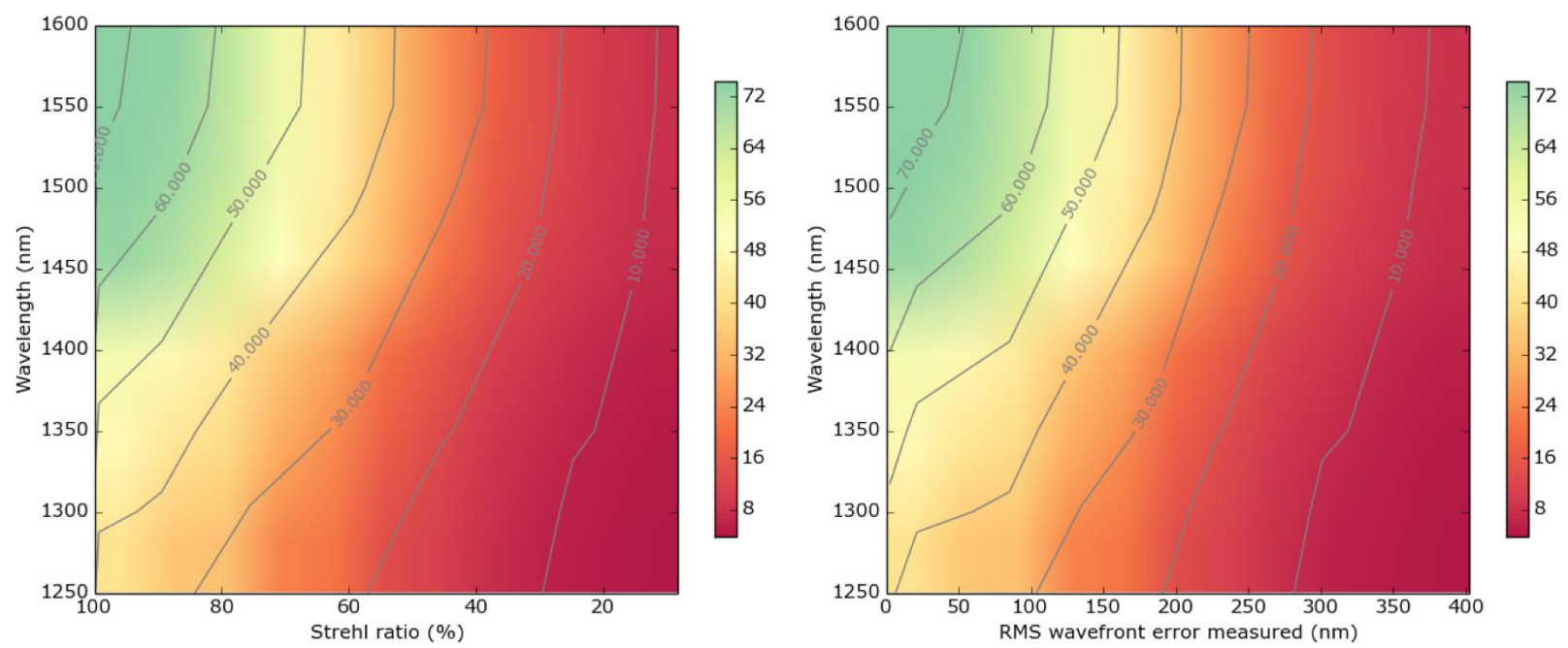

Figure 4. (Left) Coupling efficiency as a function of the Strehl ratio. (Right) Coupling efficiency as a function of the applied RMS wavefront error.

(i.e. the Strehl is near 100\%). Note, given the pupil geometry for Subaru Telescope, the theoretical maximum coupling efficiency of a non-apodized beam would be $59 \%$, which clearly demonstrates the benefit of the PIAA optics for coupling.

The higher coupling at the longer wavelengths is attributed to the fact the system was optimized for coupling at $1550 \mathrm{~nm}$ where the spot size best matches the mode size and the lower wavefront errors at longer wavelengths. At other wavelengths the spot size and mode size diverge reducing the coupling. It can also be seen that as the Strehl ratio is reduced (i.e. the wavefront error increases) the coupling efficiency decreases in a monotonic way. This validates that the optimum coupling into a SMF occurs when the wavefront is flat (i.e. $0 \mathrm{~nm}$ RMS wavefront error) and decreases the further one diverges from this condition.

The maximum theoretical coupling into a SMF using PIAA lenses is $\sim 99 \%$. However, if one considers that light is diffracted away from the PSF due to the spiders supporting the secondary mirror, the theoretical maximum coupling efficiency drops to $91 \%$. Therefore the result above demonstrates the potential to obtain $81 \%$ of the theoretical maximum achievable coupling efficiency in practice. The non-ideal coupling efficiency could be attributed to several factors or a combination thereof, including: a non-perfect focal ratio of injection, non-common wavefront error between the internal NIR camera and the fiber injection as well as imperfect PIAA optics. The final point is most likely the main contributor to the reduced coupling and will be the subject of further investigation. Nonetheless, assuming ExAO levels of wavefront correction $(80 \mathrm{~nm}$ RMS wavefront error at $1600 \mathrm{~nm}$ ) it is clear to see that the predicted coupling efficiency is $\sim 65 \%$. This demonstrates that with ExAO levels of wavefront correction it is possible to obtain coupling efficiency's of the order of that seen in MMF-fed spectrographs.

The coupling efficiency was subsequently measured on-sky on the night of the $18^{\text {th }}$ of March, 2015 on Alpha Boo. Data was acquired around UT2 : 26 hrs. 3 data sets of $15 \mathrm{~s}$ length, sampled at $1 \mathrm{kHz}$ were collected in each of the 6 spectral channels with the SMF and MMF respectively. Data sets with no light falling on the detector were also collected to subtract off the bias. The mean coupling efficiency over the $15 \mathrm{~s}$ time interval is plotted for one data set in the left panel of Fig. 5. The coupling efficiency reaches a maximum of $24 \%$ at $1500 \mathrm{~nm}$ and is dominated by residual wavefront error from uncorrected turbulence. The right panel in Fig. 5 shows the corresponding $15 \mathrm{~s}$ temporal sequence of the coupling efficiency for the $1550 \mathrm{~nm}$ spectral channel. It is clear that the coupling in fact has a strong modulation. Indeed at some point in the time series the coupling almost vanishes. The structure of the temporal sequence is indicative of a periodic perturbation. A power spectral density plot of this data is shown in Fig. 6. The power spectrum reveals a strong modulation at $6.3 \mathrm{~Hz}$. By employing accelerometers mounted on the top ring of the telescope, it was determined that this was associated with strong telescope vibrations which manifest as tip/tilt errors. This is a known issue with the Subaru Telescope and the 

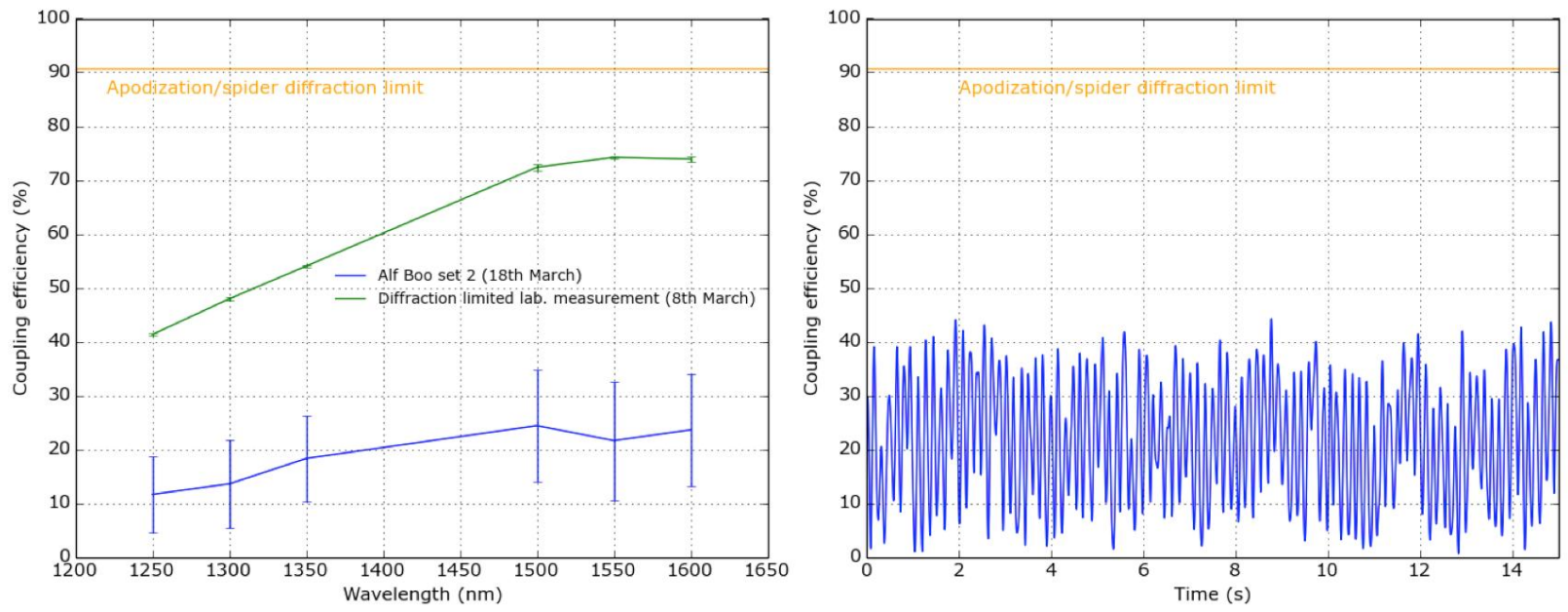

Figure 5. Coupling efficiency into a SMF on the target Alpha (Alf) Boo, on the $18^{\text {th }}$ of March 2015. (Left) Coupling efficiency as a function of wavelength (Right) Coupling efficiency as a function of time at $1550 \mathrm{~nm}$.

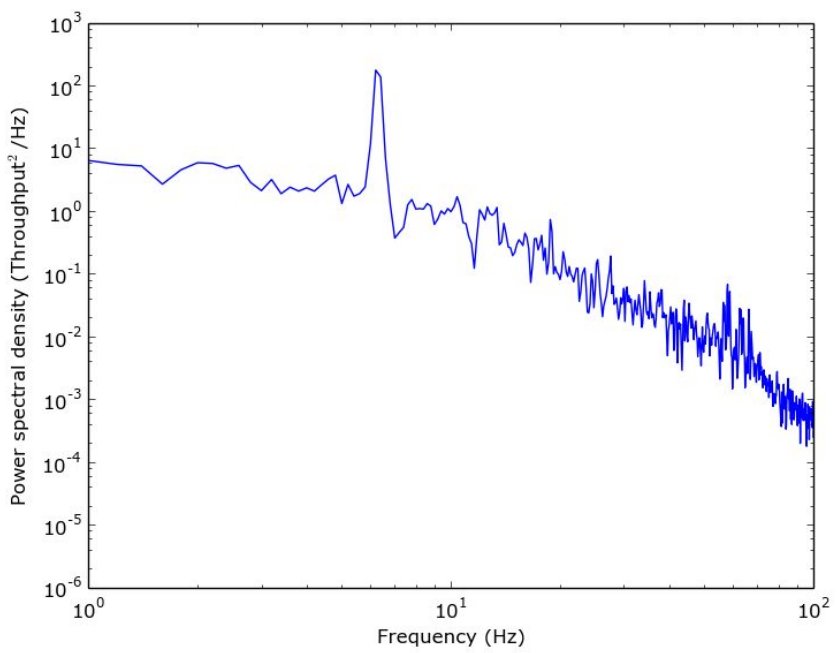

Figure 6. Power spectral density of the coupling efficiency time sequence.

SCExAO control loop is currently being modified in order to try and measure and eliminate this by employing a linear, quadratic, Gaussian (LQG) control loop. ${ }^{14}$ Alternatively one could observe in periods outside the times with high levels of vibration.

On the night of $21^{\text {st }}$ of March, 2015, data was acquired on the target Alpha Hydrae at UT7 : 00 hrs. The Strehl ratio behind SCExAO was much higher (65-70\%) on this target as there were no telescope vibrations and the seeing was better than median (0.5" in v-band). The coupling efficiency is shown in Fig. 7 for a single data set and is unsurprisingly better than for Alpha Boo. It can be seen that the mean coupling efficiency reaches $48 \%$ at 1500-1550 $\mathrm{nm}$. The temporal plot (right panel) shows that the coupling even gets up to $60 \%$ at some points and it is the large occasional dips in coupling that bring down the mean. The temporal signature has no noticeable structure to it like it did for Alpha Boo which is consistent with our choice of a period free from telescope vibrations. By using the $48 \%$ coupling efficiency it is possible to determine the Strehl ratio indirectly from the left panel in Fig. 4. It can be seen that this level of coupling at $1550 \mathrm{~nm}$ would correspond to $65-70 \%$ Strehl ratio which is consistent with that determined from focal plane images.

As the wavefront control on SCExAO improves throughout commissioning, and the residual wavefront error is further reduced, the coupling efficiency will steadily increase to the levels observed in the laboratory. The encouraging result is that the laboratory characterization of the coupling as a function of turbulence settings is 

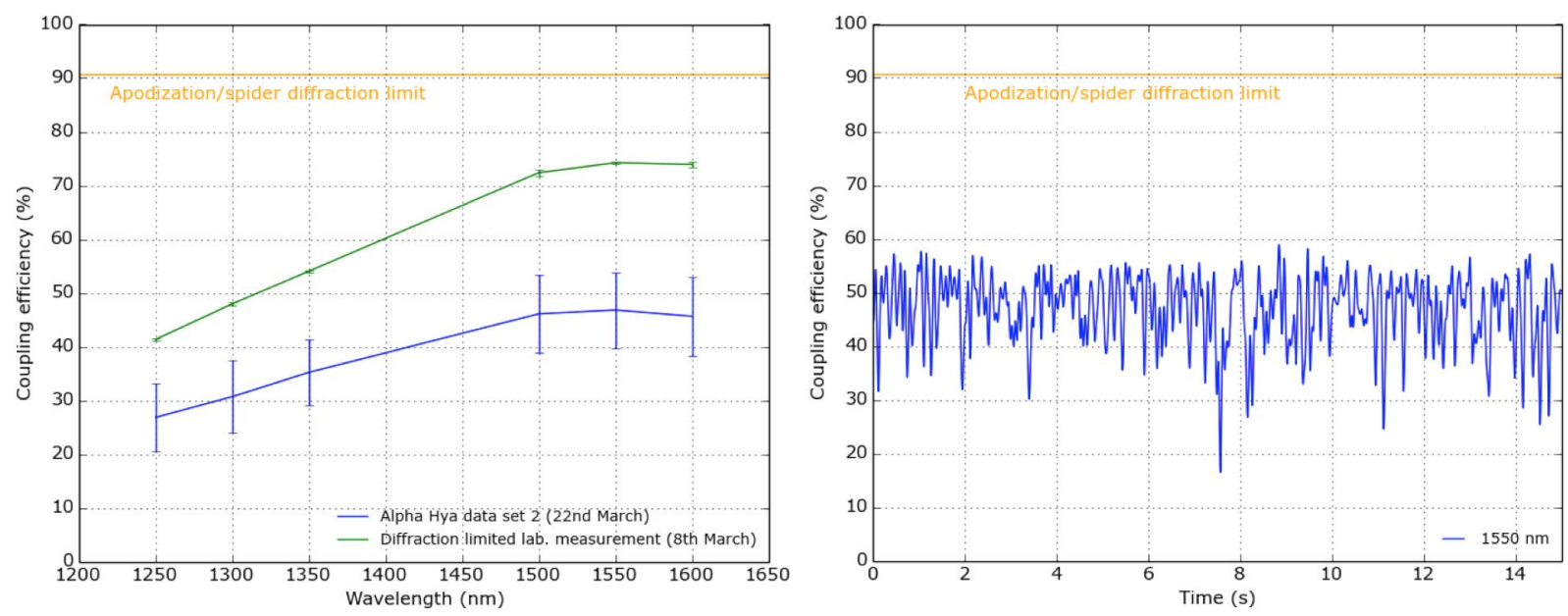

Figure 7. Coupling efficiency into a SMF on the target Alpha Hya, on the $21^{\text {st }}$ of March 2015. (Left) Coupling efficiency as a function of wavelength (Right) Coupling efficiency as a function of time at $1550 \mathrm{~nm}$.

useful for predicting on-sky levels of performance.

\subsection{SMF fed spectroscopy}

The photonic spectrograph had a $44 \pm 3 \%$ efficiency as measured from the SMF to the detector plane (not including the camera QE) for an unpolarized beam at $1550 \mathrm{~nm}$. It offered a $R>4500$ at the center of each FSR. The spectrograph was operated on numerous observing nights in the background of normal SCExAO operation and some preliminary data is shown in Fig. 8. The data was taken on the night of the $29^{\text {th }}$ of July, 2015 on the target MuCep. The left panel shows a co-add consisting of 89 cubes, with 100 frames each which were dark subtracted, flat fielded and destriped. The exposure time was $200 \mathrm{~ms}$. The J-H gap is clearly visible. The right panel shows an extracted spectrum from such an image. A template spectrum taken from IRTF's catalog is shown for reference. ${ }^{15}$ It is clear that the extracted spectrum closely resembles the reference spectrum validating the successful operation of the photonic spectrograph. The extracted spectrum suffers a little from poor wavelength calibration and flat fielding which can be remedied in future to improve the quality of the data.
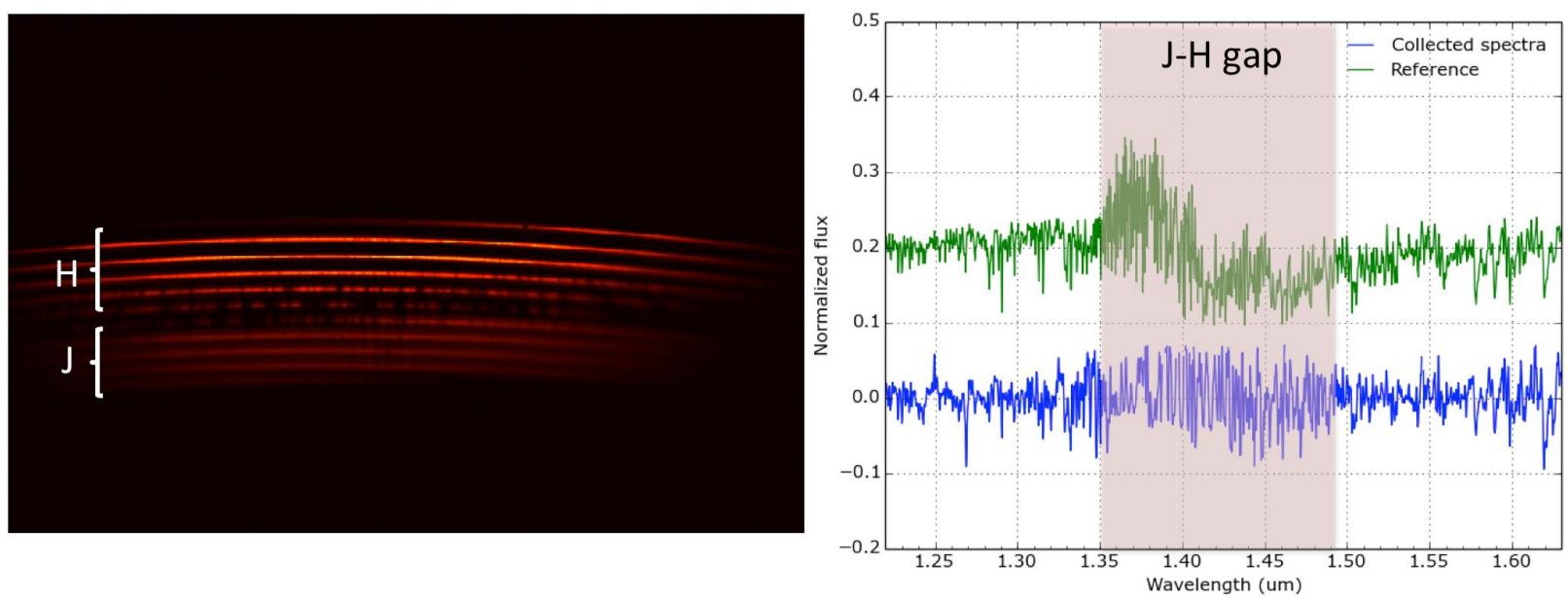

Figure 8. (Left) Co-added image of a spectrum taken with the photonic spectrograph. (Right) Extracted spectrum from MuCep compared to a reference spectrum. Reference spectrum is vertically offset for easier comparison. 


\section{DISCUSSION}

This work shows the first comprehensive study of the coupling efficiency into a SMF from a telescope while exploiting apodization optics and advanced wavefront control. The peak coupling efficiency observed was $74 \%$ in the diffraction limit, which is $\sim 15 \%$ higher than the theoretical limit for coupling to a SMF from Subaru telescope given the large secondary mirror. This unequivocally validates that the PIAA lenses are useful for fiber injection. In addition, a map of coupling efficiency as a function of the degree of wavefront correction was built and will be useful for predicting future on-sky performance.

With $65 \%$ Strehl ratio in the H-band on-sky, a coupling efficiency of $48 \%$ was obtained and with improvements to the control loop of SCExAO is forecast to increase on brighter targets ( $i<9^{\text {th }}$ magnitude). This efficient demonstration of coupling to a SMF was subsequently exploited to conduct low resolution spectroscopy. A spectrum of the star MuCep was presented validating the highly efficient (44\%) photonic-based spectrograph and demonstrating one potential application of a SMF fiber injection behind an ExAO system.

\section{ACKNOWLEDGMENTS}

This research was supported by the Australian Research Council Centre of Excellence for Ultrahigh bandwidth Devices for Optical Systems (project number CE110001018). The authors acknowledge support from the JSPS (Grant-in-Aid for Research \#23340051 \& \#26220704).

\section{REFERENCES}

[1] Bland-Hawthorn, J., Englund, M., and Edvell, G., "New approach to atmospheric OH suppression using an aperiodic fibre Bragg grating," Optics Express 12, 5902 (2004).

[2] Cvetojevic, N., Lawrence, J. S., Ellis, S. C., Bland-Hawthorn, J., Haynes, R., and Horton, A., "Characterization and on-sky demonstration of an integrated photonic spectrograph for astronomy," Optics Express 17, 18643-18650 (Oct. 2009).

[3] Coudé du Foresto, V., "Integrated optics in astronomical interferometry [invited]," in [Very High Angular Resolution Imaging], Robertson, J. G. and Tango, W. J., eds., IAU Symposium 158, 261 (1994).

[4] Spaleniak, I., Jovanovic, N., Gross, S., Ireland, M. J., Lawrence, J. S., and Withford, M. J., "Integrated photonic building blocks for next-generation astronomical instrumentation II: the multimode to single mode transition," Optics Express 21, 27197 (Nov. 2013).

[5] Wizinowich, P., Acton, D. S., Shelton, C., Stomski, P., Gathright, J., Ho, K., Lupton, W., Tsubota, K., Lai, O., Max, C., Brase, J., An, J., Avicola, K., Olivier, S., Gavel, D., Macintosh, B., Ghez, A., and Larkin, J., "First Light Adaptive Optics Images from the Keck II Telescope: A New Era of High Angular Resolution Imagery," PASP 112, 315-319 (Mar. 2000).

[6] Minowa, Y., Hayano, Y., Oya, S., Watanabe, M., Hattori, M., Guyon, O., Egner, S., Saito, Y., Ito, M., Takami, H., Garrel, V., Colley, S., Golota, T., and Iye, M., "Performance of Subaru adaptive optics system AO188," in [Adaptive Optics Systems II], Proc. SPIE 7736, 77363N (July 2010).

[7] Macintosh, B., Graham, J. R., Ingraham, P., Konopacky, Q., Marois, C., Perrin, M., Poyneer, L., Bauman, B., Barman, T., Burrows, A. S., Cardwell, A., Chilcote, J., De Rosa, R. J., Dillon, D., Doyon, R., Dunn, J., Erikson, D., Fitzgerald, M. P., Gavel, D., Goodsell, S., Hartung, M., Hibon, P., Kalas, P., Larkin, J., Maire, J., Marchis, F., Marley, M. S., McBride, J., Millar-Blanchaer, M., Morzinski, K., Norton, A., Oppenheimer, B. R., Palmer, D., Patience, J., Pueyo, L., Rantakyro, F., Sadakuni, N., Saddlemyer, L., Savransky, D., Serio, A., Soummer, R., Sivaramakrishnan, A., Song, I., Thomas, S., Wallace, J. K., Wiktorowicz, S., and Wolff, S., "First light of the Gemini Planet Imager," Proceedings of the National Academy of Science 111, 12661-12666 (2014).

[8] Vigan, A., Bonnefoy, M., Ginski, C., Beust, H., Galicher, R., Janson, M., Baudino, J.-L., Buenzli, E., Hagelberg, J., D’Orazi, V., Desidera, S., Maire, A.-L., Gratton, R., Sauvage, J.-F., Chauvin, G., Thalmann, C., Malo, L., Salter, G., Zurlo, A., Antichi, J., Baruffolo, A., Baudoz, P., Blanchard, P., Boccaletti, A., Beuzit, J.-L., Carle, M., Claudi, R., Costille, A., Delboulbé, A., Dohlen, K., Dominik, C., Feldt, M., Fusco, T., Gluck, L., Girard, J., Giro, E., Gry, C., Henning, T., Hubin, N., Hugot, E., Jaquet, M., Kasper, M., Lagrange, A.-M., Langlois, M., Le Mignant, D., Llored, M., Madec, F., Martinez, P., Mawet, D., Mesa, 
D., Milli, J., Mouillet, D., Moulin, T., Moutou, C., Origné, A., Pavlov, A., Perret, D., Petit, C., Pragt, J., Puget, P., Rabou, P., Rochat, S., Roelfsema, R., Salasnich, B., Schmid, H.-M., Sevin, A., Siebenmorgen, R., Smette, A., Stadler, E., Suarez, M., Turatto, M., Udry, S., Vakili, F., Wahhaj, Z., Weber, L., and Wildi, F., "First light of the VLT planet finder SPHERE. I. Detection and characterization of the substellar companion GJ 758 B," A $\& A$ 587, A55 (Mar. 2016).

[9] Guyon, O., "Phase-induced amplitude apodization of telescope pupils for extrasolar terrestrial planet imaging," A $\mathscr{G A}$ 404, 379-387 (June 2003).

[10] Jovanovic, N., Martinache, F., Guyon, O., Clergeon, C., Singh, G., Kudo, T., Garrel, V., Newman, K., Doughty, D., Lozi, J., Males, J., Minowa, Y., Hayano, Y., Takato, N., Morino, J., Kuhn, J., Serabyn, E., Norris, B., Tuthill, P., Schworer, G., Stewart, P., Close, L., Huby, E., Perrin, G., Lacour, S., Gauchet, L., Vievard, S., Murakami, N., Oshiyama, F., Baba, N., Matsuo, T., Nishikawa, J., Tamura, M., Lai, O., Marchis, F., Duchene, G., Kotani, T., and Woillez, J., "The Subaru Coronagraphic Extreme Adaptive Optics System: Enabling High-Contrast Imaging on Solar-System Scales," PASP 127, 890-910 (Sept. 2015).

[11] Lozi, J., Martinache, F., and Guyon, O., "Phase-Induced Amplitude Apodization on Centrally Obscured Pupils: Design and First Laboratory Demonstration for the Subaru Telescope Pupil," PASP 121, 1232-1244 (Nov. 2009).

[12] Cvetojevic, N., Jovanovic, N., Lawrence, J., Withford, M., and Bland-Hawthorn, J., "Developing arrayed waveguide grating spectrographs for multi-object astronomical spectroscopy," Optics Express 20, 2062 (Jan. 2012).

[13] Cvetojevic, N., Jovanovic, N., Betters, C., Lawrence, J. S., Ellis, S. C., Robertson, G., and Bland-Hawthorn, J., "First starlight spectrum captured using an integrated photonic micro-spectrograph," $A \mathscr{E} A \mathbf{5 4 4}$, L1 (Aug. 2012).

[14] Lozi, J., Guyon, O., Jovanovic, N., Singh, G., Goebel, S., and Okita, H., "Characterizing and mitigating vibrations for SCExAO," in [Adaptive Optics Systems V], Proc. SPIE 9909, 9909-19 (July 2016).

[15] Rayner, J. T., Cushing, M. C., and Vacca, W. D., "The Infrared Telescope Facility (IRTF) Spectral Library: Cool Stars," ApJS 185, 289-432 (Dec. 2009). 